\title{
Output stream of binding neuron with instantaneous feedback
}

\author{
A.K. Vidybida ${ }^{a}$ \\ Bogolyubov Institute for Theoretical Physics, Metrologichna str. 14-B, 03680 Kyiv, Ukraine
}

Eur. Phys. J. B 65, 577 (2008)

DOI : $10.1140 /$ epjb/e2008-00360-1

Received 27 March 2009

Published online 22 April 2009 - (C) EDP Sciences, Società Italiana di Fisica, Springer-Verlag 2009

Both in the caption of Figures 5 and 6 , measuring units $\mathrm{s}^{-1}$ should be replaced with $\mathrm{ms}^{-1}$, as follows. Right panel in Figure 6 appeared incorrect due to confused units. The correct panel is displayed below. Notice the remarkable similarity between BNF and leaky integrate and fire model in Figure 6.
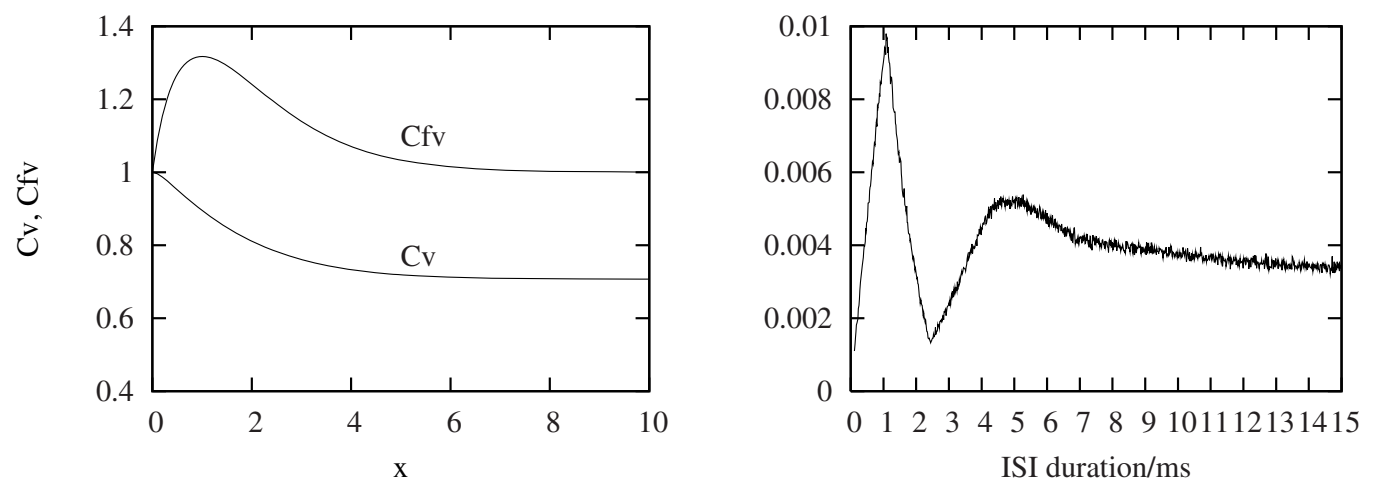

Fig. 5. Left: Coefficient of variation as function of $x=\lambda \tau$ for $\mathrm{BN}(\mathrm{Cv})$ and $\mathrm{BNF}(\mathrm{Cfv})$. Right: ISI distribution $P_{b}(t)$ (measured in $\mathrm{ms}^{-1}$ ) found numerically for leaky integrate and fire model with feedback. Used 30000000 output spikes. Firing threshold, $C=20 \mathrm{mV}$, input intensity, $\lambda=0.1 \mathrm{~ms}^{-1}$, membrane time constant, $\tau_{M}=6 \mathrm{~ms}$, input impulse amplitude, $y_{0}=7.5 \mathrm{mV}$.
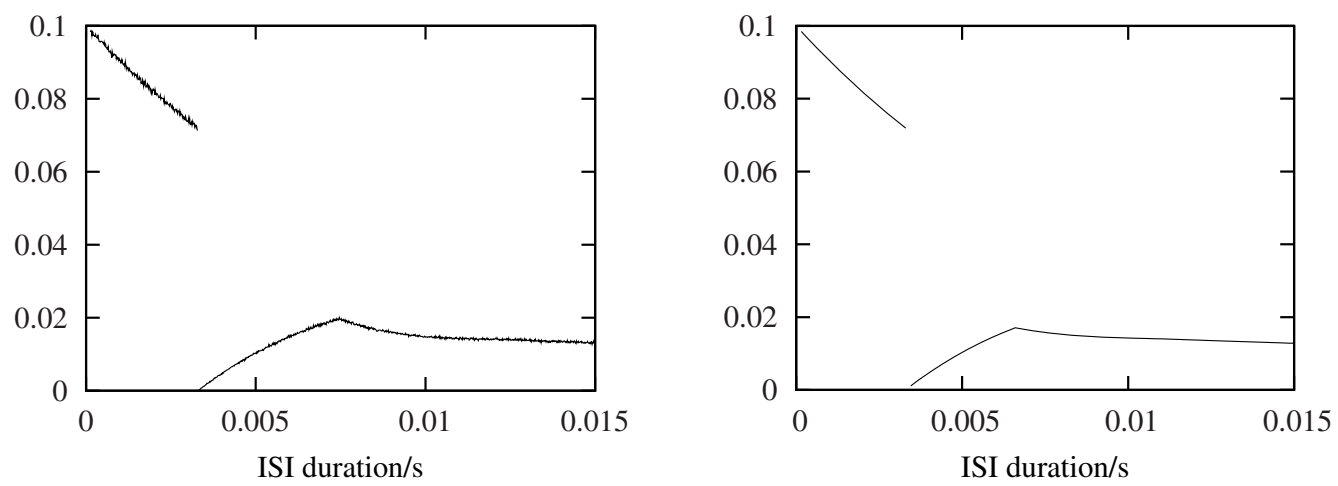

Fig. 6. Left: ISI distribution $P_{b}(t)$ found numerically for leaky integrate and fire model with feedback. Used 30000000 output spikes. Firing threshold, $C=20 \mathrm{mV}$, input intensity, $\lambda=0.1 \mathrm{~ms}^{-1}$, membrane time constant, $\tau_{M}=3 \mathrm{~ms}$, input impulse amplitude, $y_{0}=15 \mathrm{mV}$. Right: BNF ISI distribution in accordance with equations (4) and (7) displayed for comparison. Here $\lambda=0.1 \mathrm{~ms}^{-1}, \tau=3.3 \mathrm{~ms}$. Both densities are measured in $\mathrm{ms}^{-1}$. Compare also with Figure 3 .

\footnotetext{
a e-mail: vidybida@bitp.kiev.ua
} 